\title{
Transmittance of MIM Waveguides Containing Semi- Circular Ring Shaped and Rectangular Ring Shaped Grooves
}

\author{
Naser Hatefi-Kargan and Milad Zare-Zardini \\ Department of Physics, University of Sistan and Baluchestan, Zahedan, Iran \\ *Corresponding author Email: n.hatefi@phys.usb.ac.ir
}

Received: Nov. 3, 2016, Revised: Dec. 6, 2016, Accepted: Dec. 19, 2016, Available Online: Oct. 28, 2017

\begin{abstract}
In this paper the transmittance of metal-insulator-metal (MIM) waveguides containing semi-circular ring shaped and rectangular ring shaped grooves are investigated theoretically in the $500 \mathrm{~nm}$ to 1700 nm wavelength range. By varying groove parameters their effects on the transmittance of the MIM waveguides are investigated. The results show that the transmittance spectra of the waveguides have maximum and minimum points, and by using appropriate parameters for the grooves it is possible to make the transmittance maximum or minimum at a required frequency. Therefore by using the ring shaped grooves in the MIM waveguides a frequency splitter is designed. The results show that the efficiency of an MIM waveguide containing the ring shaped groove for filtering a required frequency is higher than the efficiency of an MIM waveguide containing tooth shaped groove.
\end{abstract}

KEYWORDS: MIM waveguide, Plasmonic filter, Plasmonic waveguide, Surface plasmon.

\section{INTRODUCTION}

Surface plasmon polaritons (SPPs) are electromagnetic excitations, i.e. electromagnetic waves coupled to the free electrons of a conductor, propagating along the interface of a dielectric and a conductor. These waves are confined to the interface evanescently in perpendicular direction [1]. The metal-insulator-metal (MIM) waveguides are the simple and primary types of waveguides that support propagation of SPPs with acceptable propagation length. The propagation length of the SPPs in MIM waveguides are limited due to loss in metallic layers, and techniques have been proposed to enhance their propagation length [2]. Owing to their wide applications these waveguides have been considered by many research groups [3]. Many structures based on MIM waveguides have been investigated such as: $U$ shaped waveguide [4], Y shaped combiners [5], frequency filters and splitters [6,7], MachZehender interferometer [8] and plasmonic absorption switches [9]. Owing to the fact that surface plasmon waves are confined to the metal-dielectric interface, refractive index sensors [10], DNA sequencing structures [11], plasmonic nanoloop arry antenas [12] have been proposed and investigated theoretically or experimentally. Various structures for filtering an optical wavelength in plasmonic structures have been proposed and studied such as: a tooth shaped frequency filter which is an MIM waveguide with a rectangular groove [13], a brag reflector which is an MIM waveguide containing rectangular grooves with different heights [14], the MIM waveguides with triangular grooves [15], and the trapezoidal resonator waveguide [16]. In these structures, groove dimensions and the material inside the groove are important, because variation in the dimensions and material affect the effective index of the groove, and the amount of the power entered into the groove [17]. In general, these structures work based on the phase difference 
between the wave propagating inside the MIM waveguide in straight direction and the part propagating inside the groove [18].

In the reported MIM waveguide structures containing grooves for filtering purposes [1316], the grooves interact through one edge with the wave propagating inside the MIM waveguide in the straight direction. It is expected that if grooves are designed so that the grooves interact with the wave through two edges, then the efficiency would be enhanced. For this purpose two new structures for filtering SPPs are proposed, and their transmittance at different frequencies are investigated. These structures are the MIM waveguides with semi-circular ring shaped and rectangular ring shaped grooves. By varying groove dimensions, the effect of groove parameters affecting the transmittance of the waveguides has been investigated. Also by using semi-circular ring shaped grooves a frequency splitter has been designed which directs the required wavelength at a desired direction.

\section{Simulation Model}

The proposed waveguides have been shown in Fig. 1. In this figure the yellow colored areas are metal (silver) and the white ones are dielectric (air). Normally a function based on the Drude model is used for the dielectric constant of silver [19]. In order to improve the results, in this paper experimentally measured data reported by Johnson and Christy [20] has been interpolated and used as the dielectric function of silver. Dielectric constant of air is one. Figure 1(a) shows an MIM waveguide with a semi-circular ring shaped groove. In order to the average length of the semi-circular ring shaped groove to be Leff (the length of dashed line inside the groove) and its thickness to be $w_{s}$, then in Fig. 1(a) it is required that $r_{s}=\left(L_{\text {eff }} / \pi-\mathrm{w}_{s} / 2\right)$ and $r_{1}=\left(L_{\text {eff }} / \pi+\mathrm{w}_{\mathrm{s}} / 2\right)$.

Figure 1(b) shows an MIM waveguide with a rectangular ring shaped groove. For calculating transmittance, the power incident on the port $\mathrm{A}$ and the power passing through the port B are calculated. The ports are placed at a distance of ' $d$ ' from the grooves. A part of the SPPs propagating in the waveguide when reaches to the first edge of the semi-circular ring shaped groove or rectangular ring shaped groove, enters into the groove and the remained part propagates in the straight direction. The part of the wave propagating inside the groove travels longer distance than the part traveling in the straight direction. Therefore when both parts reach to the second edge of the groove there will be a phase difference between them. If the phase difference is even numbers of $\pi$, they will interfere constructively and the transmittance will be high, but if the phase difference is odd numbers of $\pi$, they will interfere destructively and the SPPs wave will not propagate through the waveguide and will be reflected back. This behavior causes the transmittance spectrum of an MIM waveguide containing the groove to have maximum and minimum points.

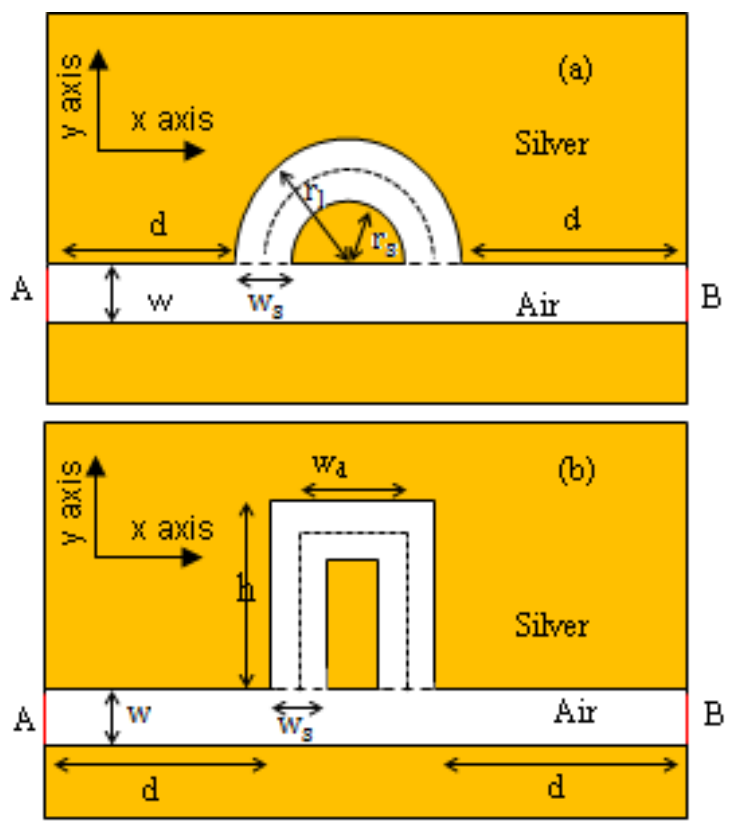

Fig. 1. Structural geometry of an MIM waveguide containing, (a) a semi-circular and (b) rectangular ring shaped groove.

For simulating the waveguides shown in Fig. 1 , the Helmholtz equation,

$\nabla \times\left(\varepsilon_{r}+i \varepsilon_{i}\right)^{-1}(\nabla \times \mathbf{H})-k_{0}^{2} \mu_{r} \mathbf{H}=0$ 
is used by applying perfectly matched layers boundary condition at the outer boundaries of the waveguides, ensuring the absorption of waves reaching to the outer boundaries without any reflection. In Eq. 1, $\mu_{r}$ is the relative permeability, $\varepsilon_{r}$ is the real part of relative permittivity, $\varepsilon_{i}$ is the imaginary part of relative permittivity, $\varepsilon_{0}$ is the free space permittivity, $\mathbf{H}$ is the magnetic field vector of the electromagnetic wave, and $k_{0}=\omega / c$, where $\omega$ is the angular frequency of the wave propagating inside the waveguide and $c$ is the speed of light in free space. In driving Eq. 1 by using Maxwell's curl equations it is assumed that the fields are harmonic with $e^{-i \omega t}$ time dependence. For doing simulations, it is assumed that in Fig. 1 a TM polarized wave (Magnetic field $\mathbf{H}$ of the wave in $\mathbf{z}$ direction, i.e. perpendicular to paper surface) with frequency $\omega$ excites the entrance of the waveguide (Port A). Then the propagation of the wave inside waveguide is simulated by using Eq.1, where finite element method is used with using a commercial software. The TM wave is used because only TM waves can propagate in MIM plasmonic waveguides [1].

\section{III.SiMULATION RESULTS}

Figure 2(a) shows the transmittance spectrum of the waveguide shown in Fig. 1(a) when $L_{\text {eff }}=200 \mathrm{~nm}, \mathrm{w}=50 \mathrm{~nm}, d=100 \mathrm{~nm}$, and $\mathrm{w}_{s}=50$ $\mathrm{nm}$. As the figure shows the transmittance of the waveguide when $L$ eff $=200 \mathrm{~nm}$ is negligible for the wavelength of $519 \mathrm{~nm}$, and the maximum of transmittance with a value of $79.5 \%$ is at the wavelength of $868 \mathrm{~nm}$. Figure 2(b) shows the transmittance of the semicircular ring shaped groove itself as a function of $L_{\text {eff }}$ at $\lambda=868 \mathrm{~nm}$, where the transmittance is maximum. The transmittance of the groove is oscillatory as a function of Leff with a general trend of decrease, which is due to the increase in loss with the increase of path length. The oscillatory nature of the transmittance with $L_{\text {eff }}$ is due to the reflectance from the both ends of the groove and the standing waves that form inside the groove at appropriate values of $L_{\text {eff. }}$.
To validate this point, an MIM waveguide is considered with the same width and length as the semi-circular groove. The simulations show that for $\lambda=868 \mathrm{~nm}$ the SPPs wave propagate inside the waveguide with an effective index of $n_{\text {eff }}=1.32$. If the waveguide is considered as a Fabry-Perot resonator, then the resonances occur when $L_{\text {eff }}=m \lambda / 2 n_{\text {eff }}$ where $m$ is a positive integer and $\lambda$ is the wavelength of the wave in free space. For a fixed value of $\lambda$ two adjacent resonance peaks occur when $\Delta L_{\text {eff }}=\lambda / 2 n_{\text {eff }}$ where for $\lambda=868 \mathrm{~nm} \quad \Delta L_{\text {eff }}=328.79 \mathrm{~nm}$ is calculated. From Fig. 2(b) the distance between two maximum points is $\Delta L_{\text {eff }}=320.18 \mathrm{~nm}$ which is in good agreement with the value obtained by using the Fabry-Perot resonance peak points.
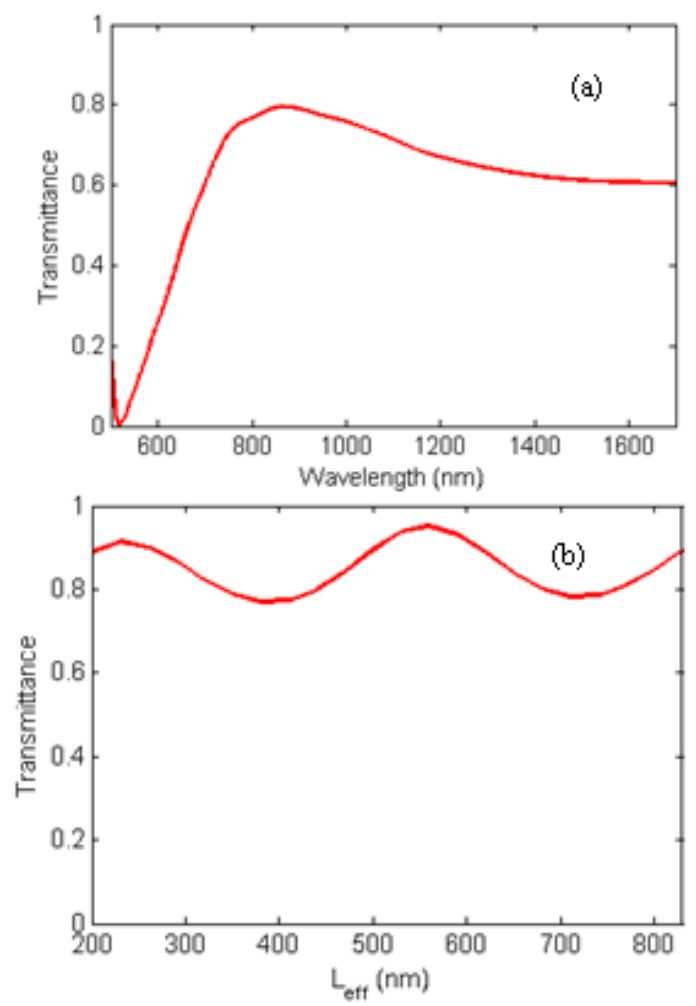

Fig. 2. (a) Transmittance spectrum of the waveguide shown in Fig. 1(a) where $\mathrm{w}=50 \mathrm{~nm}$, $d=100 \mathrm{~nm}, L_{\text {eff }}=200 \mathrm{~nm}$, and $\mathrm{w}_{s}=50 \mathrm{~nm} ;$ (b) transmittance of the semi-circular ring shaped groove itself as a function of $L_{\text {eff }}$ at $\lambda=868 \mathrm{~nm}$.

Figure 3 shows the transmittance of the waveguide shown in Fig. 1(b) for $h=200 \mathrm{~nm}$, 
$d=100 \mathrm{~nm}, \mathrm{w}=50 \mathrm{~nm}, \mathrm{w}_{d}=100 \mathrm{~nm}$, and $\mathrm{w}_{s}=50$ $\mathrm{nm}$. In this case there is an absolute transmittance maximum with a value of $84.5 \%$ at $\lambda=610 \mathrm{~nm}$ and two minimum points at $\lambda=738 \mathrm{~nm}$ and $\lambda=1022 \mathrm{~nm}$. Results of simulations, not presented here, show that similar to the transmittance of semi-circular ring shaped groove, the transmittance of rectangular ring shaped groove itself at a fixed value of wavelength varies oscillatory with a general trend of decrease as ' $h$ ' and ' $w$ ' increase.

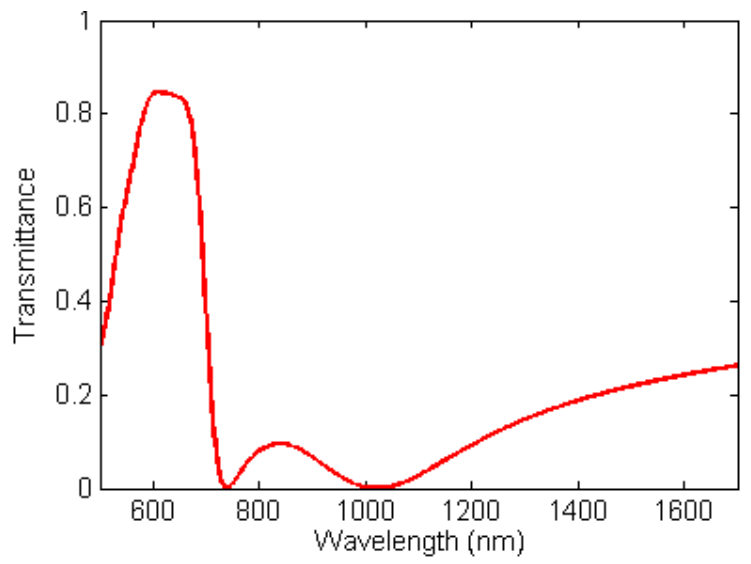

Fig. 3. Transmittance spectrum of the waveguide shown in Fig. 1(b) where $\mathrm{w}=50 \mathrm{~nm}, d=100 \mathrm{~nm}$, $h=200 \mathrm{~nm}, \mathrm{w}_{d}=100 \mathrm{~nm}$, and $\mathrm{w}_{s}=50 \mathrm{~nm}$.

Figure 4 shows the energy density inside the waveguide shown in Fig. 1(a) for $\lambda=519 \mathrm{~nm}$ and $\lambda=868 \mathrm{~nm}$ where the transmittance spectrum is maximum and minimum respectively. As the figure shows at $\lambda=519$ $\mathrm{nm}$ the wave does not propagate after the semi-circular ring shaped groove, while the wave propagates after the groove at $\lambda=868$ $\mathrm{nm}$. Figure 5 shows such a behavior for the waveguide shown in Fig. 1(b) at $\lambda=610 \mathrm{~nm}$ and $\lambda=738 \mathrm{~nm}$ where the transmittance spectrum is maximum and minimum respectively.

In the following sections the effect of the semi-circular ring shaped and the rectangular ring shaped groove parameters on the transmittance of MIM waveguides containing the grooves are investigated.
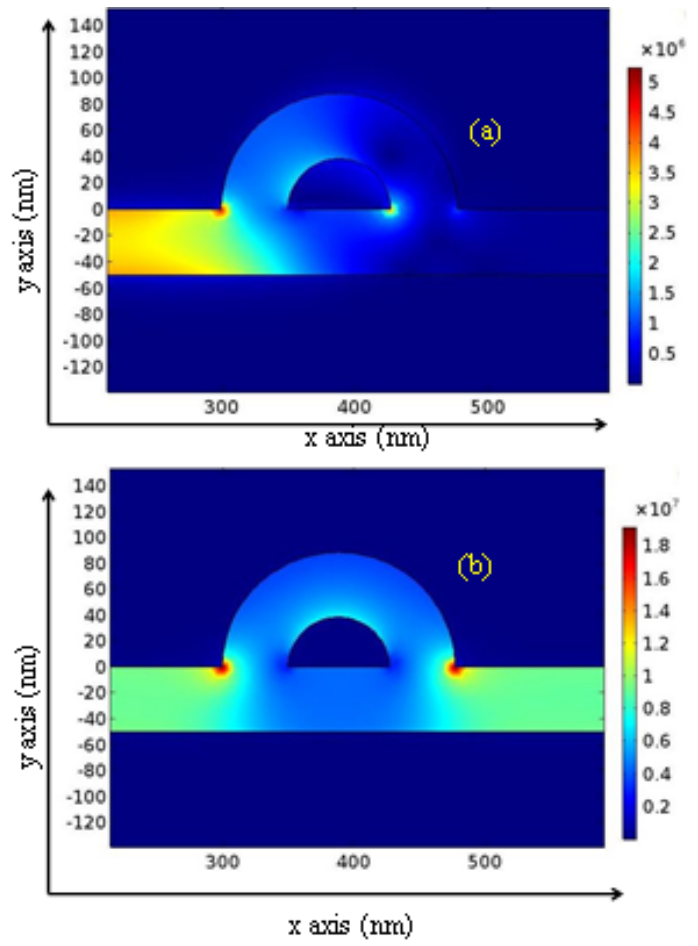

Fig. 4. Energy density inside the waveguide shown in Fig. 1(a) where $L_{\text {eff }}=200 \mathrm{~nm}, \mathrm{w}=50 \mathrm{~nm}, d=100$ $\mathrm{nm}$, and $\mathrm{w}_{s}=50 \mathrm{~nm}$ for the wavelengths: (a) $\lambda=519 \mathrm{~nm}$, and (b) $\lambda=868 \mathrm{~nm}$.
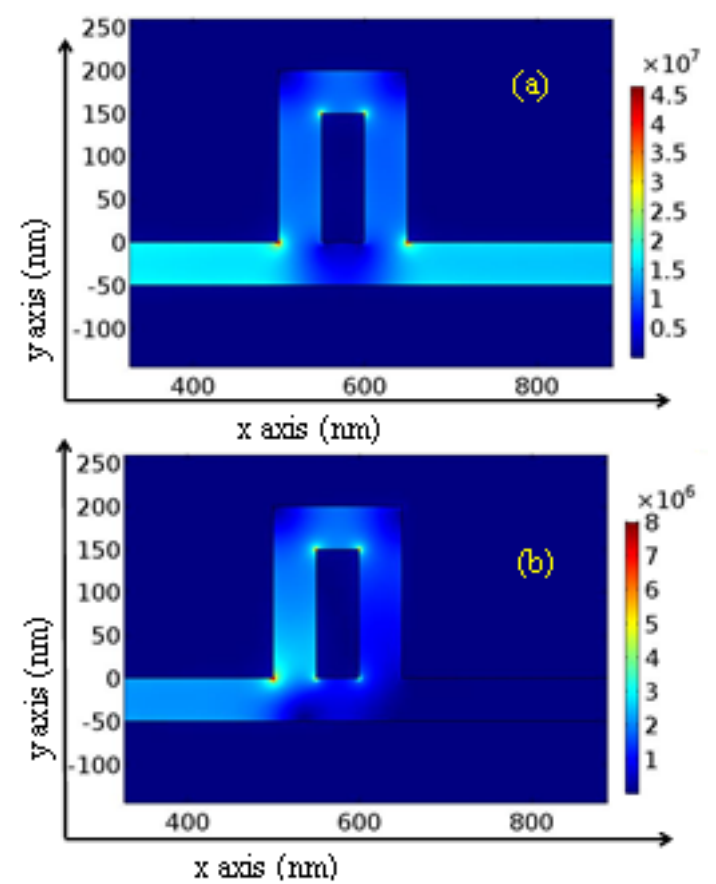

Fig. 5. Energy density inside the waveguide shown in Fig. 1(b) where $\mathrm{w}=50 \mathrm{~nm}, d=100 \mathrm{~nm}, h=200$ $\mathrm{nm}, \mathrm{w}_{d}=100 \mathrm{~nm}$, and $\mathrm{w}_{s}=50 \mathrm{~nm}$ for the wavelengths: (a) $\lambda=610 \mathrm{~nm}$, and (b) $\lambda=738 \mathrm{~nm}$. 


\section{A. The Effect of Semi-Circular Ring Shaped Groove Parameters on Transmittance}

In order to investigate the effect of radius of semi-circular ring shaped grooves on transmittance, $L_{\text {eff }}$ in Fig. 1(a) is varied. Figure 6 shows the transmittance spectra of the structure shown in Fig. 1(a) for different values of $L_{\text {eff }}$ where $\mathrm{w}=50 \mathrm{~nm}, d=100 \mathrm{~nm}$ and $\mathrm{W}_{s}=50 \mathrm{~nm}$. As the figure shows with the increase of $L_{\text {eff, }}$, the transmittance spectrum is shifted toward longer wavelengths.

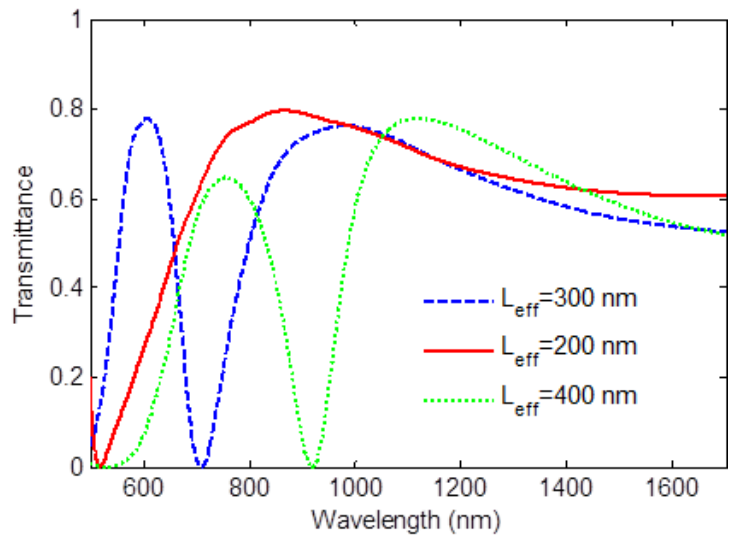

Fig. 6. Transmittance spectra of the waveguide shown in Fig. 1(a) for $L_{\text {eff }}=200 \mathrm{~nm}, L_{\text {eff }}=300 \mathrm{~nm}$, and $L_{\text {eff }}=400 \mathrm{~nm}$, where $d=100 \mathrm{~nm}, \mathrm{w}=50 \mathrm{~nm}$, and $\mathrm{W}_{s}=50 \mathrm{~nm}$.

Varying the thickness of semi-circular ring shaped groove varies the effective refractive index of the groove which varies the wavelength of light inside the groove and the amount of the power entered into the groove. Therefore the transmittance of the groove is as a result of variation in both effective index and the amount of power entered into the groove. Figure 7 shows the transmittance spectra of the waveguide shown in Fig. 1(a) at $\mathrm{w}_{s}=25 \mathrm{~nm}$ and $\mathrm{w}_{s}=50 \mathrm{~nm}$, where $L_{\text {eff }}=300 \mathrm{~nm}, \mathrm{w}=50 \mathrm{~nm}$, and $d=100 \mathrm{~nm}$. As the figure shows the value of transmittance at maximum points increase with the increase of the thickness of the semicircular ring shaped groove. The reason is that with the increase of the thickness, the loss of the groove decreases. In order to investigate this point, the transmittance of the semicircular ring shaped groove itself was calculated at two different thicknesses. The transmittance was $57 \%$ for $\mathrm{w}_{s}=25 \mathrm{~nm}$ and $83 \%$ for $\mathrm{w}_{s}=50 \mathrm{~nm}$, indicating that with the increase of thickness, loss of the groove decreases.

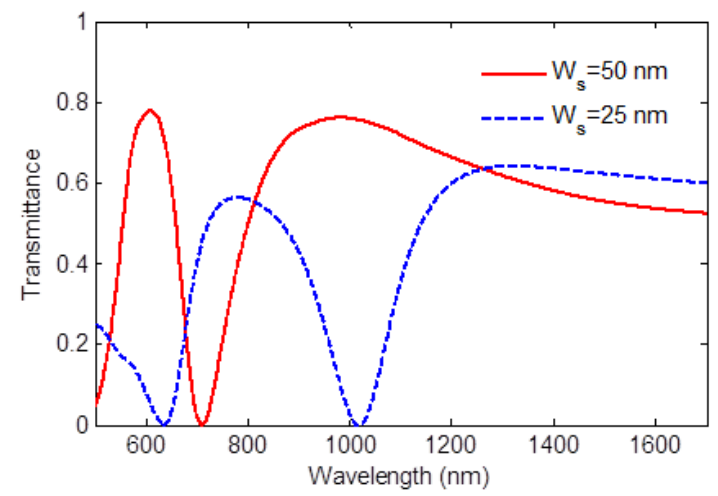

Fig. 7. Transmittance spectra of the waveguide shown in Fig. 1(a) for $\mathrm{w}_{s}=25 \mathrm{~nm}$ and $\mathrm{w}_{s}=50 \mathrm{~nm}$ where $d=100 \mathrm{~nm}, L_{\text {eff }}=300 \mathrm{~nm}$, and $\mathrm{w}=50 \mathrm{~nm}$.

\section{B. The Effect of Rectangular Ring Shaped Groove Parameters on Transmittance}

Figure 8 shows the effect of the height of rectangular ring shaped groove $(h)$ on the transmittance of an MIM waveguide containing the groove. With the increase of ' $h$ ' the path difference between the wave travelling inside the groove and the wave travelling in straight direction increases, so the maximum and minimum points on the transmittance spectrum are shifted toward longer wavelengths. In addition with the increase of ' $h$ ' the values of transmittance at maximum points increase as well. The reason is that the transmittance of the rectangular ring shaped groove itself as a function of ' $h$ ' has an oscillatory nature, similar to the one shown in Fig. 2(b) for semi-circular ring shaped groove, and for the ranges of ' $h$ ' considered in this study it is on the increasing part of the curve which overcomes the loss due to metallic layers with increasing path length.

If in Fig. 1(b) the width $(\mathrm{wd})$ of the rectangular ring is varied, then the path difference between the wave propagating inside the groove and the part of the wave propagating in straight direction will increase which makes the transmittance spectrum to vary. Figure 9 
shows such a variation where with the increase of ' $\mathrm{Wd}_{\mathrm{d}}$ ' the spectrum is shifted toward longer wavelengths.

Figure 10 shows the transmittance spectra of the waveguide shown in Fig. 1(b) when the thickness of the rectangular ring $\left(\mathrm{w}_{s}\right)$ is varied. With the increase of ' $\mathrm{w} s$ ' the effective index of the ring decreases, so the optical path difference between the wave inside the ring and the wave propagating in straight direction decrease as ' $\mathrm{W}$ ' ' increases. Therefore in this case with the increase of ' $\mathrm{w}_{s}$ ' the transmittance spectrum is shifted toward shorter wavelengths.

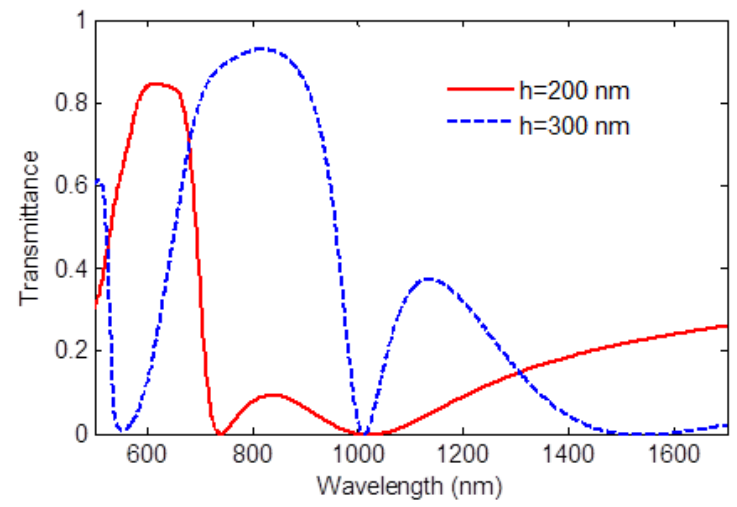

Fig. 8. Transmittance spectra of the waveguide shown in Fig. 1(b) for $h=200 \mathrm{~nm}$, and $h=300 \mathrm{~nm}$, where $\mathrm{w}=50, \mathrm{w}_{d}=100 \mathrm{~nm}, \mathrm{w}_{s}=50 \mathrm{~nm}$, and $d=100$ $\mathrm{nm}$.

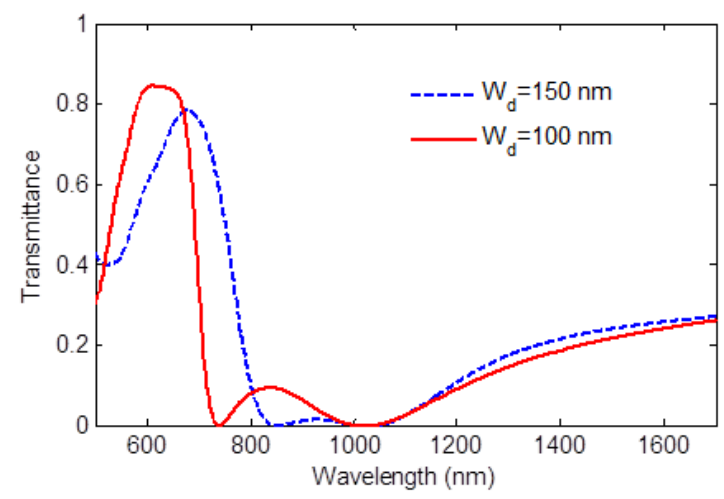

Fig. 9. Transmittance spectra of the waveguide shown in Fig. 1(b) for $\mathrm{w}_{d}=100 \mathrm{~nm}$, and $\mathrm{w}_{d}=150$ $\mathrm{nm}$, where $d=100 \mathrm{~nm}, \mathrm{w}=50 \mathrm{~nm}, \mathrm{w}_{s}=50 \mathrm{~nm}$, and $h=200 \mathrm{~nm}$.

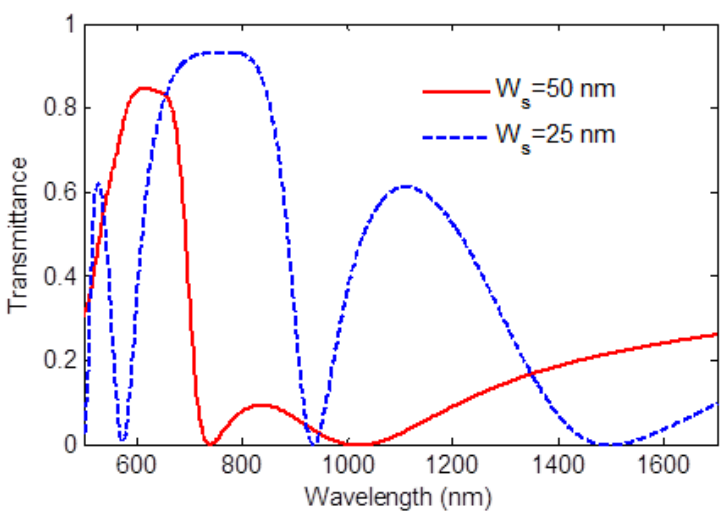

Fig. 10. Transmittance spectra of the waveguide shown in Fig. 1(b) for $\mathrm{w}_{s}=50 \mathrm{~nm}$, and $\mathrm{w}_{s}=25 \mathrm{~nm}$, where $d=100 \mathrm{~nm}, \mathrm{w}=50 \mathrm{~nm}, \mathrm{w}_{d}=100 \mathrm{~nm}$, and $h=200 \mathrm{~nm}$.

\section{IV.TRANSMitTANCE OF ARRAY OF GROOVES}

In this section the effect of a few grooves in an MIM waveguide on the transmittance of the waveguide is investigated. Figure 11 shows an MIM waveguide with three semi-circular ring shaped grooves. The transmittance of this waveguide when $d=100 \mathrm{~nm}, \mathrm{w}=50 \mathrm{~nm}$, and $L_{\text {eff }}=300 \mathrm{~nm}$ was calculated, the transmittance was maximized at maximum points when the distance between the semi-circular rings was equal to ' $w$ '.

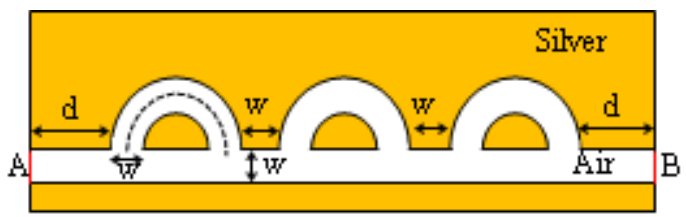

Fig. 11. An MIM waveguide with three semicircular ring shaped grooves, where $d=100 \mathrm{~nm}$, $\mathrm{w}=50 \mathrm{~nm}$ and effective length of each semi-circular ring is $L_{\text {eff }}=300 \mathrm{~nm}$.

At the points where the transmittance of an MIM waveguide with one semi-circular ring shaped groove is zero, the transmittance of the MIM waveguide with numbers of semicircular ring shaped grooves become zero in a broad range of wavelengths around those points, as shown in Fig. 12. The same effect was observed for the transmittance of an MIM 
waveguide with numbers of rectangular ring shaped grooves.

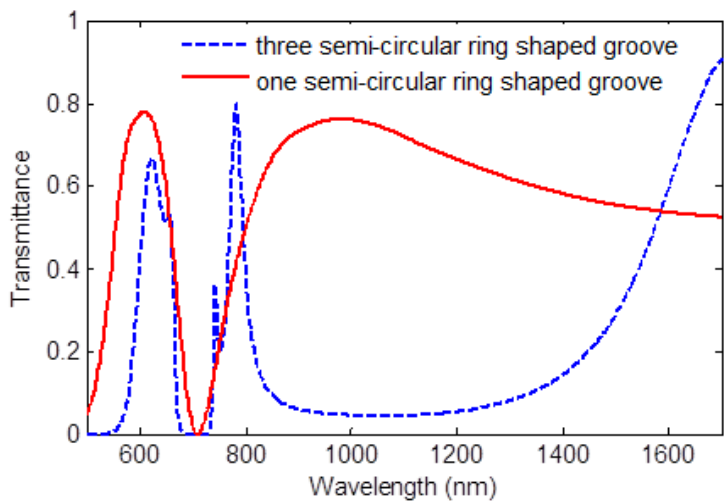

Fig. 12. Transmittance spectra of the waveguide shown in Fig. 11 with one and three semi-circular ring shaped grooves.

\section{Designing Splitter BASEd ON RING SHAPED GROOVES}

As was studied in previous sections the transmittance of an MIM waveguide containing ring shaped grooves becomes zero at some wavelengths, and by choosing appropriate parameters for the structure of grooves it is possible to design an appropriate MIM waveguide so that to transmit or block a required wavelength. Figure 13 shows a Tshaped waveguide containing two semicircular ring shaped grooves. If for example $L_{\text {eff }}=290 \mathrm{~nm}$ for the groove on the left side and $L_{\text {eff }}=364 \mathrm{~nm}$ for the groove on the right side of Fig. 13, then the left side of the Tshaped waveguide will block the wave with $\lambda=710 \mathrm{~nm}$ while transmitting the wave with $\lambda=570 \mathrm{~nm}$, and the right side will block the wave with $\lambda=570 \mathrm{~nm}$ while transmitting the wave with $\lambda=710 \mathrm{~nm}$. Therefore the Tshaped waveguide in Fig.13 can split these two wavelengths. Figure 14 shows the results of simulations where the left side of T-shaped waveguide transmits $\lambda=570 \mathrm{~nm}$ and the right side transmits the $\lambda=710 \mathrm{~nm}$.

By choosing appropriate parameters it is possible to design the same splitter with rectangular ring shaped grooves as well.

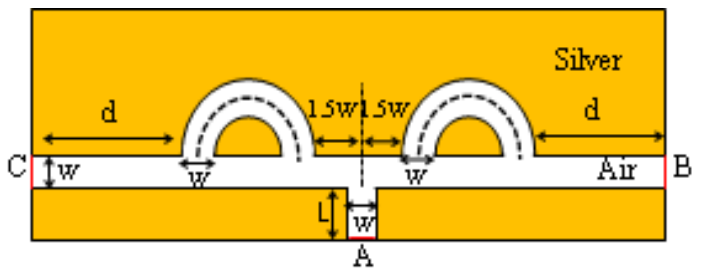

Fig. 13. T-shaped MIM waveguide with two semicircular ring shaped grooves.
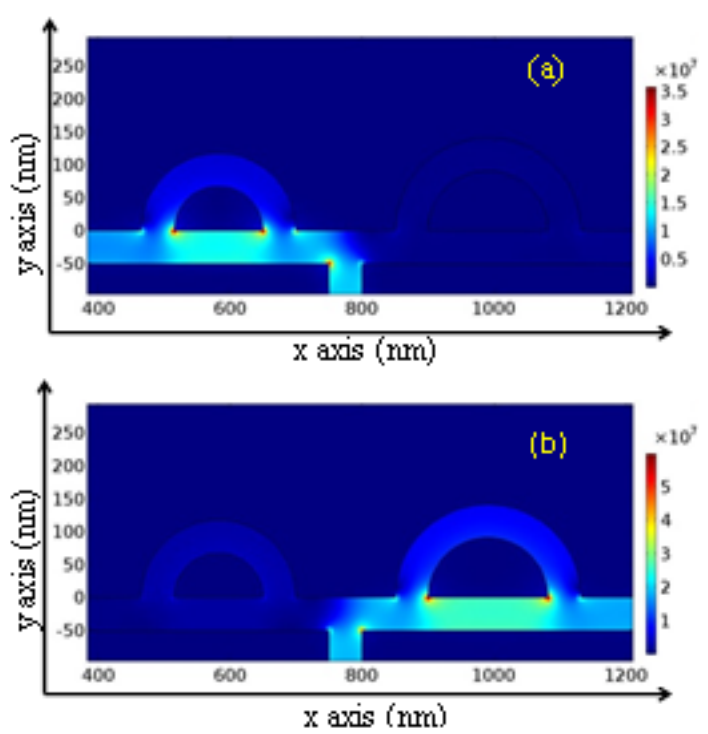

Fig. 14. Energy density inside the T-shaped waveguide for the SPPs waves with wavelengths: (a) $\lambda=570 \mathrm{~nm}$, and (b) $\lambda=710 \mathrm{~nm}$ for the waveguide shown in Fig. 13 where $\mathrm{w}=50 \mathrm{~nm}$, and the effective length of semi-circular ring shaped grooves on the left and right sides are respectively: $L_{\text {eff }}=290 \mathrm{~nm}$, and $L_{\text {eff }}=364 \mathrm{~nm}$.

\section{COMPARING MIM WAVEGUIDES WITH DIFFERENT GROOVES}

Figure 15 shows an MIM waveguide containing a tooth shaped groove. The MIM waveguide with tooth shaped groove has been studied in reference [13]. The advantage of MIM waveguides containing the ring shaped grooves relative to tooth shaped groove is that for a required wavelength to have minimum transmittance the MIM waveguides containing ring shaped grooves, as was studied in previous sections, work more efficiently than the MIM waveguide containing tooth shaped groove. For example if in Fig. 1 and in Fig. 15 $d=100 \mathrm{~nm}, \mathrm{w}=50 \mathrm{~nm}$, and $\mathrm{w}_{s}=50 \mathrm{~nm}$ are chosen, and the required wavelength for 
minimum transmittance is $\lambda=570 \mathrm{~nm}$, then it is required that for the waveguide shown in Fig. 1(a) Leff $=300 \mathrm{~nm}$, for the one shown in Fig. 1(b) $\mathrm{w}_{d}=100 \mathrm{~nm}$ and $h=189 \mathrm{~nm}$, and for the waveguide shown in Fig. $15 h=353 \mathrm{~nm}$ are chosen. With these parameters the reflectance of the waveguides shown in Fig. 1(a), Fig. 1(b) and Fig. 15 are $93 \%, 93.7 \%$ and $86 \%$ respectively, so the MIM waveguides with ring shaped grooves reflect the required wavelength much more than the MIM waveguide with tooth shaped groove. This indicates that ring shaped grooves block the transmission of a required wavelength more efficiently.

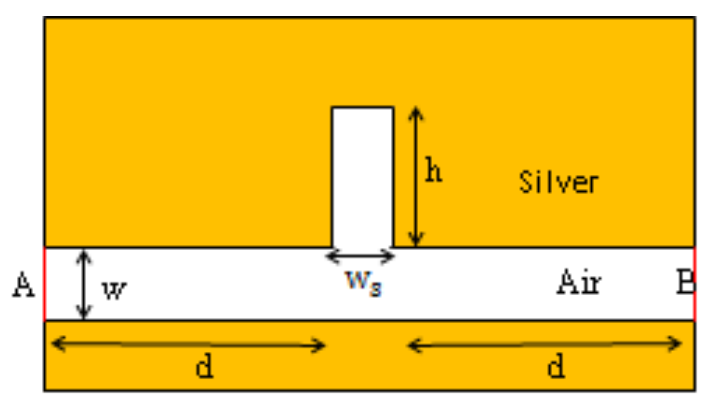

Fig. 15. An MIM waveguide with a tooth shaped groove.

\section{CONCLUSION}

The transmittances of MIM waveguides containing semi-circular ring shaped and rectangular ring shaped grooves were studied. The transmittances are zero at some wavelengths, and more than $90 \%$ at some other wavelengths. This indicates that these waveguides can be used as filters. The structural parameters of the grooves affect the optical path difference between the part of the wave propagating inside the grooves and the part propagating in straight direction. Therefore the wavelengths of maximum and minimum points on the transmittance spectra depend on the grooves structural parameters. By using this fact a frequency splitter based on a T-shaped MIM waveguide containing semicircular ring shaped grooves has been designed. Comparing the obtained results show that MIM waveguides containing semi- circular and rectangular ring shaped grooves have higher performance on filtering surface plasmon waves relative to MIM waveguides containing tooth shaped grooves. Therefore the MIM waveguides containing ring shaped grooves can be used for fabricating efficient wavelength splitters.

\section{ACKNOWLEDGMENT}

This work was supported by the University of Sistan and Baluchestan.

\section{REFERENCES}

[1] S.A. Maier, Plasmonic: Fundamentals and Applications, New York: Springer, 2007.

[2] M.A. Izadi and R. Nouroozi, "Non-degenerate optical parametric amplification analysis of surface plasmon polariton wave in a silver coated PPLN planar waveguide," Superlattice. Microst., Vol. 100, pp. 431-439, 2016.

[3] G. Veronis and S. Fan, "Bends and splitters in subwavelength metal-dielectric-metal plasmonic waveguides," Appl. Phys. Lett., Vol. 87, pp. 131102 (1-3), 2005.

[4] T.W. Lee and S.K. Gray, "Subwavelength light bending by metal slit structures," Opt. Express, Vol. 13, pp. 9652-9659, 2005.

[5] H. Gao, H. Shi, C. Wang, C. Du, X. Luo, Q. Deng, Y. Lv, X. Lin, and H. Yao, "Surface plasmon polariton propagation and combination in Y-shaped metallic channels," Opt. Express, Vol. 13, pp. 10795-10800, 2005.

[6] Q. Zhang, X.G. Huang, X.S Lin, J. Tao, and X.P. Jin, "A subwavelength coupler-type MIM optical filter," Opt. Express, Vol. 17, pp. 7549-7554, 2009.

[7] R.A. Wahsheh, Z. Lu, and M.A.G. Abushagur, "Nanoplasmonic couplers and splitters," Opt. Express, Vol. 17, pp. 19033-19040, 2009.

[8] Z. Han, L. Liu, and E. Forsberg, "Ultracompact directional couplers and MachZehnder interferometers employing surface plasmon polaritons," Opt. Commun., Vol. 259, pp. 690-695, 2006.

[9] C. Min and G. Veronis, "Absorption switches in metal-dielectric-metal plasmonic 
waveguides," Opt. Express, Vol. 17, pp. 10757-10766, 2009.

[10] J. Homola, Surface Plasmon Resonance Based Sensors, Berlin: Springer-Verlag, 2006.

[11]B. Fotouhi, V. Ahmadi, and V. Faramarzi, "Nano-plasmonic-based structures for DNA sequencing," Opt. Lett., Vol. 41, pp. 42294232, 2016.

[12] A. Ahmadi and H. Mosallaei, "Plasmonic nanoloop array antenna, " Opt. Lett., Vol. 35, pp. 3706-3708, 2010.

[13] Y. Matsuzaki, T. Okamoto, M. Haraguchi, M. Fukui, and M. Nakagaki, "Characteristics of gap plasmon waveguide with stub structures," Opt. Express, Vol. 16, pp. 16314-16325, 2008.

[14] J. Tao, X.G. Huang, X. Lin, Q. Zhang, and X. Jin, "A narrow-band subwavelength plasmonic waveguide filter with asymmetrical multiple-teeth-shaped structure," Opt. Express, Vol. 17, pp. 13989-13994, 2009.

[15] S.M. Ebadi, S. Salman Sajjadi, M. Sajjad Bayati, and S. Bonyadi Ram, "A novel plasmonic high pass wavelength filter based on triangular arrays in a MIM waveguide," IEEE Photonics North Conf., June 9-11, 2015.

[16] C. Song, S. Qu, J. Wang, B. Tang, X. Xia, X. Liang, and $\mathrm{Y}$. $\mathrm{Lu}$, "Plasmonic tunable filter based on trapezoid resonator waveguide," J. Mod. Optic., Vol. 62, pp. 1400-1404, 2015.

[17]L. Sun, J. Wang, Y. Wang, H. Liu, C. Liu, and S. Gao, "Electromagnetically induced transparency of double-groove shaped plasmonic waveguide," Optik, Vol. 126, pp. 2596-2599, 2015.

[18] X. Gao and L. Ning, "Optical switch effect of metal-dielectric-metal plasmonic waveguide coupled with stub structure," Optik, Vol. 123, pp. 1326-1328, 2012.

[19] G. Shvets and I. Tsukerman, Plasmonics and Plasmonic Metamaterials: Analysis and
Applications, World Scientific Publishing Co. Pte. Ltd., 2012.

[20] P.B. Johnson and R.W. Christy, "Optical constants of the noble metals," Phys. Rev. B, Vol. 6, pp. 4370-4379, 1972.

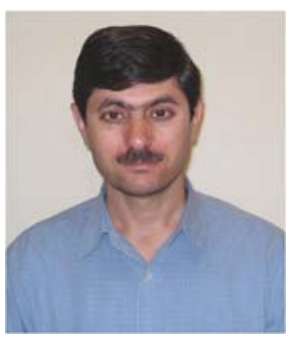

Naser Hatefi-Kargan recievied his $\mathrm{PhD}$ degree from the University of Leeds in 2007. $\mathrm{He}$ is now with the Physics Department of the University of Sistan and Baluchestan as an assistant professor. Dr. Hatefi-Kargan is a member of both Optics and Photonics Society and the Physics Society of Iran. His research areas are semiconductor photonic, and plasmonic devices.

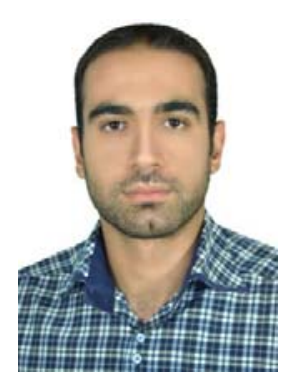

Milad Zare-Zardini received his BSc degree in Electronic Engineering form Yazd University in 2013, and his MSc degree in Photonics from the University of Sistan and Baluchestan in 2016. His research interest is analysis and simulation of plasmonic devices. 


\section{THIS PAGE IS INTENTIONALLY LEFT BLANK.}

